\title{
Echocardiography: An everyday tool in intensive care
}

Muhammad Fuad Bangash, MD, FCCP, Chief, Pulmonary\& Critical Care, Medical Director, ICU, Steward Norwood Hospital, Norwood, MA 02062, USAE-mail: lifesavericu@icloud.com

It was a cold December night in Minneapolis, Minnesota. I was in ICU taking care of a patient, Mr. David, who had had a cardiac arrest at home, underwent $\mathrm{CPR}$ and was now on mechanical ventilation. He had been subjected to 'Mild Induced Hypothermia' protocol. The patient did well and after re-warming to normal temperature, he was weaned off sedation and mechanical ventilation. $\mathrm{He}$ was able to talk and move all his extremities. This is what we hope for in every patient after a cardiac arrest with appropriate medical care and latest technology. Patients get a second lease on life and their loved ones are able to look into their eyes and hold their hands. David's wife Mary was so happy to see him come around after she had witnessed him undergoing CPR.

Everything was going well but we were puzzled that why David's heart had stopped in the first place. Since he was stable now, we called the cardiologist, who performed cardiac catheterization, found a blockage and placed a stent.

Shortly after coming back to the ICU from the cath lab, patient became very unstable. There were signs of hypoxia and a very low blood pressure. I placed a breathing tube and started pumping IV fluids. An emergent echocardiogram confirmed collection of blood in the pericardium - there was cardiac tamponade. The cardiologist drained the blood and patient's condition became stabilized.

He had to undergo cardiac surgery to evacuate all the blood and to repair the heart vent, again to

Be kept under sedation and mechanical ventilation despite doing well earlier that day. Fortunes had changed for the worse within a few hours. David's wife was very distressed. The ICU team reassured her. Eventually, David was able to be weaned off sedation and mechanical ventilation, made a full recovery after rehab and was discharged home.

Pericardial tamponade is a 'can't-miss-it critical care catastrophe' and needs to be managed immediately. If missed, it is rapidly fatal. I had two other cases where the cause was postcardiac surgery hemorrhage and a malignant pericardial effusion.

The key signs are hypotension, tachycardia and distended jugular veins. Additional signs include a flask like silhouette on chest $\mathrm{x}$-ray, low voltage on ECG and pulsus paradoxus.

If the shock cannot be explained by history and physical examination, an emergent echocardiography will show the typical signs of pericardial effusion and RV collapse. Treatment can either be pericardial drain placement or making a pericardial window.

\section{Suspect; Act; Save}

\author{
Muhammad Fuad Bangash MD, FCCP, \\ Chief, Pulmonary\& Critical Care, Medical Director, ICU, \\ Steward Norwood Hospital, Norwood, MA 02062, USA \\ E-mail: lifesavericu@icloud.com
}

This case left a lasting mark on my memory. Susan, 72 years of age, female patient presented to ED with shortness of breath and fever. Chest x-ray showed right sided pneumonia and effusion. She was put on appropriate IV antibiotics and a small bore chest tube was placed under ultrasound guidance. Since the effusion did not drain freely a combination of tissue plasminogen activator (TPA) and dornase alpha (an inhaled drug for treating cystic fibrosis) was used by the consulting pulmonologist.

Despite appropriate antibiotic therapy and drainage, patient's condition worsened requiring mechanical ventilation and vasopressors. Patient was becoming frailer day by day due to malnutrition and also developed congestive heart failure.

Our cardiothoracic surgeon performed a video assisted decortication on the right side. Biopsy specimens were sent from the lung and pleura. The pathologist identified unusual gram positive filamentous bacteria. It was recommended that Bactrim be used IV for possible Nocardia infection. Patient condition gradually started to improve with enteral nutrition, 
diuresis, and was extubated but required a prolonged ICU stay, followed by rehabilitation.

The pleural fluid culture eventually grew Actinomyces Israeli! It was a case of a very unusual cause of pneumonia. The aggressive care with thoracic surgery, mechanical ventilation and antibiotics guided by biopsy specimens, saved Susan!
Unusual pulmonary infections are a 'Can't Miss Critical Care Catastrophes'; although rare but can prove fatal. So although our traditional approach to community acquired or healthcare associated pneumonia is enough in most patients, if they are not responding then appropriate consultations coupled with appropriate diagnostic / therapeutic procedure will save lives!

\section{IASP Early Career Research Grants Program}

Grants: Multiple grants of US\$20,000 each

Purpose: The IASP Early Career Research Grant facilitates a development of young researchers just starting their careers as independent investigators.

Timeline: The submission period for Early Career Research Grants is now closed for 2019.

Applications for 2020 will open December 13, 2019.

\section{Eligibility Requirements:}

At the time of application applicants should be:

- IASP members.

- Within 6 years of having received their final degree, or completing professional and specialty training.

- Initiating their career as an independent investigator.

\section{Grant Overview}

- IASP will award multiple grants per year to be used by eligible applicants who are starting their independent career.

- The IASP Fellowships, Grants, and Awards Working Group will make the final decisions on the awards.

- The grants are available internationally and are not related to any particular discipline.

Please contact grants@iasp-pain.org if you have any questions, or call +1-202-856-7400

https://www.iasp-pain.org/Education/GrantDetail.aspx?ItemNumber=712 